\title{
Perioperative renal failure in pediatric heart transplant recipients: Outcome and risk factors
}

Tang L, Du W, L'Ecuyer TJ. Perioperative renal failure in pediatric heart transplant recipients: Outcome and risk factors.

Pediatr Transplantation 2011: 15: 430-436. (C) 2011 John Wiley \& Sons A/S.

Abstract: PRF is encountered in $10-13 \%$ of adult heart transplants. Only one study of a single center's experience with PRF has been reported in pediatric patients. This study examines the effect of PRF on pediatric heart transplant outcome using the UNOS database. A total of 3598 patients met inclusion criteria, of whom 254 (7\%) had PRF.

The PRF group comprised 31 recipients requiring PRE and 223 recipients requiring POST. Compared with No-PRF patients, PRE patients had similar survival rate and POST patients had decreased survival rate at 30 days, one, five, and 10 -yr post-transplant $(\mathrm{p}<0.001)$. PRF patients also had significantly lower graft survival at one, five, and $10 \mathrm{yr}(\mathrm{p}<0.001)$. Risk factors for developing PRF included ECMO, ventilator, and inotropic support at listing and CHD as the listing diagnosis. PRF increased the duration of hospital stay and the incidence of chronic severe renal dysfunction. PRF that requires POST (whether or not it began pretransplant) has a significant negative impact on pediatric heart transplant outcome. Specific characteristics identify patients at particular high risk of developing PRF.

\section{Liwen Tang, Wei Du and Thomas J. L'Ecuyer}

Division of Cardiology, Children's Hospital of Michigan, Wayne State University, Detroit, MI, USA

Key words: heart transplant - renal failure

Liwen Tang, Division of Cardiology, Floating Hospital for Children, Tufts Medical Center, Tufts University, 800 Washington St, \#313, Boston, MA, 02111, USA Tel.: +617 6365067

Fax: +6176362354

E-mail: Itang@tuftsmedicalcenter.org

Accepted for publication 25 August 2010
Pediatric heart transplantation is a well-accepted treatment for children with end-stage heart disease, including cardiomyopathies, failed palliation of complex congenital heart defects, and congenital heart defects for which no effective palliation is currently available $(1,2)$. Approximately 300-400 pediatric heart transplants are performed each year in the United States. Outcomes are continually improving, with one-yr survival above $90 \%$ and five-yr survival above $78 \%(1,2)$.

PRF, defined as requiring dialysis during the period from transplant listing to discharge from the transplant hospitalization, is experienced by

\footnotetext{
Abbreviations: BOTH, pre- and post-transplant dialysis; CAD, coronary artery disease; CHD, congenital heart disease; ECMO, extracorporeal membrane oxygenation; HLHS, hypoplastic left heart syndrome; OPTN, Organ Procurement and Transplantation Network; POST, posttransplant dialysis; PRE, pretransplant dialysis; PRF, perioperative renal failure; s.d., standard deviation; SRTR, Scientific Registry of Transplant Recipients.
}

$11-15 \%$ of adult heart transplant recipients. The outcome in this group is poor, with $40 \%$ mortality before hospital discharge, more than 10 times that of recipients without PRF $(3,4)$. Risk factors for PRF in this group include older age of the recipient, hypertrophic cardiomyopathy diagnosis, the need for pretransplant ventricular assist device support, and longer donor ischemia time (5-7).

Infants and children waiting for a donor heart may have varying degrees of renal impairment, with some requiring dialysis $(8,9)$. In one single center study, 39 of $308(12.6 \%)$ transplant recipients met the definition of PRF. The one, two, and five-yr survival in the PRF group was significantly lower than that in the No-PRF group (9). Specific risk factors for developing PRF have not been identified in the pediatric heart transplant population.

The UNOS database is a multi-institutional resource that allows questions about uncommon complications in heart transplant recipients to be studied. The purpose of this study is to define the 
prevalence of PRF in pediatric cardiac transplant recipients and determine its impact on heart transplant outcome. A secondary goal of this study is to identify risk factors for developing PRF in a national pediatric heart transplant population.

\section{Methods}

We obtained national transplant data collected by the OPTN and maintained by the SRTR. OPTN has prospectively collected data on all candidates for and recipients of solid-organ transplants in the US since 1988. Data are submitted at the time of listing, at transplantation. Follow up data are collected during yearly follow up, and when significant events occur (rejection, graft failure, death, etc.). Each center must submit a yearly report for each patient that includes graft function, coronary disease, and renal function. Since 1993, the presence or absence of peri-operative renal failure, defined as requiring dialysis during the period from transplant listing to discharge from the transplant hospitalization, has been included in the registry.

We obtained data for all recipients $\leq 18$ yr of age listed for heart transplant who received a first organ between 1993 and 2008. Recipients with missing perioperative renal status and patients listed for heart transplantation who died waiting were excluded. In the UNOS/OPTN database, anonymity of patient information is maintained by coding of data during compilation, so our Institutional Review Board granted waiver of consent.

Patients were divided into two groups: (i) those with PRF and (ii) those without PRF (No-PRF). Continuous variables are summarized by mean \pm s.d. Categorical variables are shown as frequencies and percentages. Demographic data such as age, weight, height, gender, race, and diagnosis requiring transplant were compared between groups using Student's $T$-test, Fisher's exact test or chi-square test as appropriate.

Kaplan-Meier survival analysis was used to estimate one, five, and 10-yr recipient and graft survival. The impact of PRF on patient and graft survival was assessed using the stratified log-rank test. To control for illness severity, we performed the stratified log-rank test for recipients who were on ECMO support, ventilator support, and inotropic support at the time of listing. Cox proportional hazard models were used to assess risk factors for death in the PRF group. Demographic and clinical variables (medical condition, primary diagnosis, ventilator support, ECMO support, and inotropic support) were considered. Proportional-hazards assumptions were verified by assessing time-dependent covariates. Risk factors associated with developing PRF were explored in univariate and multivariate logistic regression analyses from which odds ratios and 95\% confidence intervals were generated. Variables that were significant at the 0.1 level in univariate analyses were included in a stepwise multivariate logistic regression model. Outcomes including length of hospital stay, episodes of rejection, important infections, CAD, severe renal dysfunction, and hypertension were compared between the two groups using Student's $T$-test, Fisher's exact test or chi-square test as appropriate.

\section{Results}

A total of 3712 primary pediatric cardiac transplants were performed between 1993 and 2008, of which 3598 patients had adequate data to be included and 114 recipients were excluded because of incomplete data. Seven percent (254/ 3598) met the definition for PRF. The prevalence of PRF was similar across transplant eras: 1993$1998,7.3 \%$; 1998-2003, 6.9\%; 2003-2008, 7.2\%. The PRF group comprised 254 recipients. There were 78 recipients who required PRE, of whom 47 also required POST. And there were 176 recipients required only POST.

\section{Demographic parameters}

As shown in Table 1, there was no difference between groups in age and age group. CHD was the most common primary diagnosis leading to listing for transplant in the PRF group, cardiomyopathy was the most common one in the No-PRF group. The PRF group had more Caucasian recipients than the No-PRF group. There were no significant differences between groups in weight, height, or gender.

\section{Post-transplant patient and graft survival}

Of the transplanted patients, $93.3 \%$ patients survived to 30 days post-transplant. There was a significantly lower 30-day survival in the PRF group, compared with No-PRF group ( $72 \%$ vs. $95 \% ; \mathrm{p}<0.001)$. As shown in Table 2, causes of death were similar in the PRF and No-PRF group. Early mortality data were stratified into three eras: 1993-1998; 1998-2003; and 2003-2008.

Table 1. Clinical and demographic characteristics

\begin{tabular}{lccc}
\hline & PRF $(\mathrm{n}=254)$ & No-PRF $(\mathrm{n}=3344)$ & p Value \\
\hline Age (yr) & & & \\
Mean + s.d. & $6.4(6.2)$ & $6.2(6.1)$ & 0.6 \\
Median (25th-75th) & $5(0-12)$ & $4(0-13)$ & \\
Age group (\%) & & & \\
$<1$ yr & $85(33)$ & $993(30)$ & 0.5 \\
$1-10$ yr & $117(46)$ & $1551(46)$ & 0.74 \\
$>10$ yr & $52(20)$ & $802(24)$ & 0.42 \\
Weight (kg) & $24.6(24.7)$ & $25.1(24.0)$ & \\
Height (cm) & $104(46)$ & $106(45)$ & \\
Gender (\%) & & & \\
M & $146(43)$ & $1886(56)$ & \\
F & $108(57)$ & $1458(43)$ & \\
Race (\%) & & & \\
Caucasian & $176(69)$ & $2051(61)$ & \\
Black & $36(14)$ & $634(19)$ & \\
Hispanic & $25(10)$ & $472(14)$ & \\
Other & $17(7)$ & $187(6)$ & \\
Diagnosis (\%) & $157(62)$ & $1456(44)$ & \\
CHD & $97(38)$ & $1888(56)$ & \\
Cardiomyopathy & & & \\
\hline
\end{tabular}

Clinical and demographic characteristics compared between the PRF group and No-PRF group. Asterisk denotes statistically significant difference. 
Tang et al.

Table 2. Causes of death within 30 days post-transplant

\begin{tabular}{llll}
\hline & PRF $(n=70)$ & No-PRF $(n=170)$ & $p$ Value \\
\hline Graft failure (\%) & $21(30)$ & $70(40)$ & NS \\
Infection (\%) & $15(21)$ & $13(8)$ & NS \\
Multi-organ failure (\%) & $18(26)$ & $0(0)$ & NS \\
Cardiovascular disease (\%) & $6(9)$ & $25(15)$ & NS \\
Others $(\%)$ & 0 & $62(36)$ & NS \\
\hline
\end{tabular}

Cause of death within 30 days post-transplant compared between PRF group and No-PRF group. There were no significant differences between the groups.

Early mortality in the No-PRF group decreased significantly with later era $(\mathrm{p}<0.05)$ and did decline over the transplant era. In the PRF group, however, the decrease did not reach statistical significance.
The one, five, and 10-yr patient survival rate was $45 \%, 41 \%$, and $40 \%$ in the PRF group, significantly below the No-PRF group $(89 \%$, $79 \%$, and $75 \%$; all $\mathrm{p}<0.001$ ) (Fig. 1). When survival was made conditional upon surviving to six months post-transplant, the difference between the PRF and No-PRF groups became insignificant, as shown in Fig. 2. The difference in survival between groups was therefore accounted for predominantly by early mortality.

PRF recipients were divided into three groups based upon when dialysis was required: PRE only, BOTH, and POST only. Patients' survival for each group was as follows: one yr $(97 \%$ vs. $38 \%$ vs. $38 \% ; p<0.001)$, five yr $(90 \%$ vs. $28 \%$

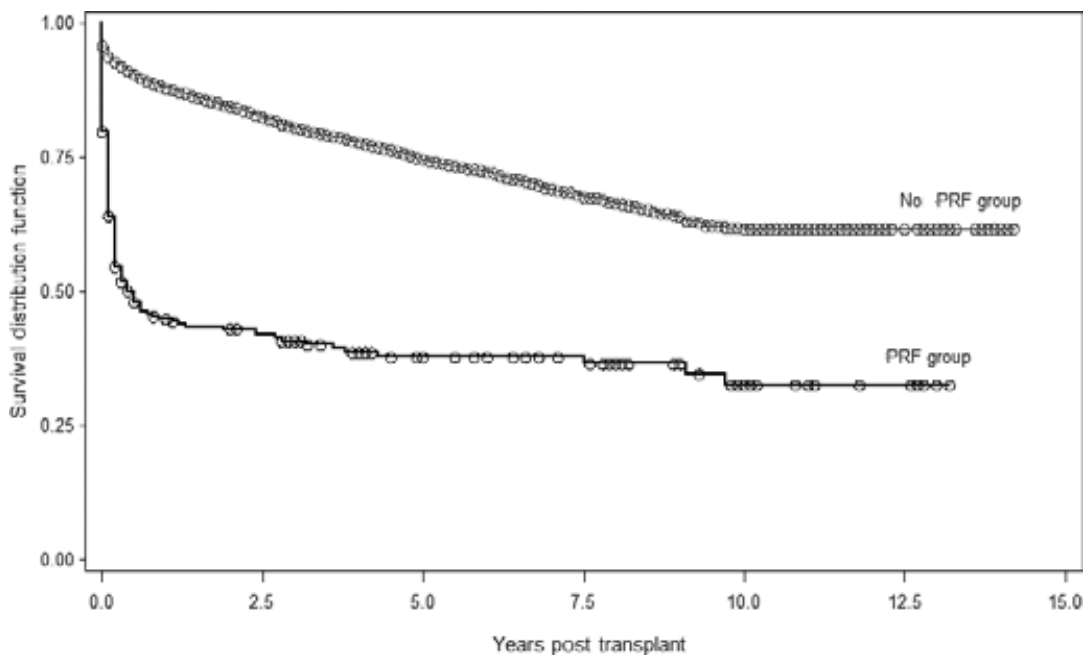

Fig. 1. Patient survival as a function of time post-transplant, stratified by presence or absence of PRF. Survival was significantly lower in the PRF than in the No-PRF group $(\mathrm{p}<0.001)$.

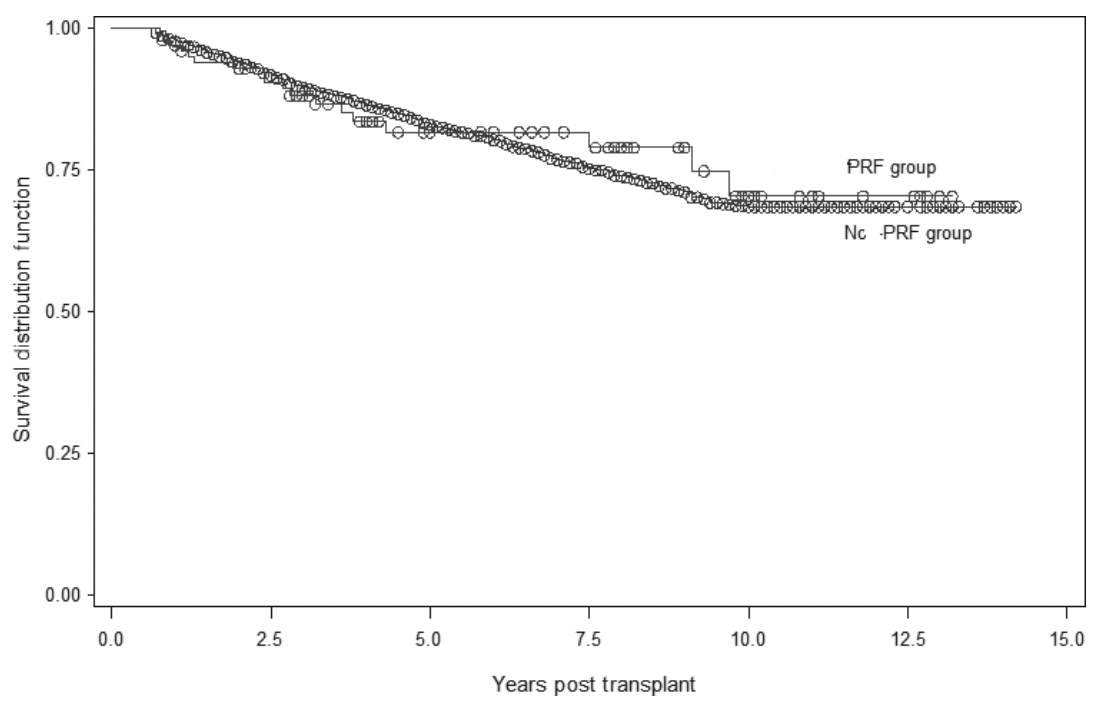

Fig. 2. Post-transplant patient survival, conditional on survival to six months, stratified by presence or absence of PRF. The survival in the PRF and No-PRF group was not significant different $(p=0.25)$. 


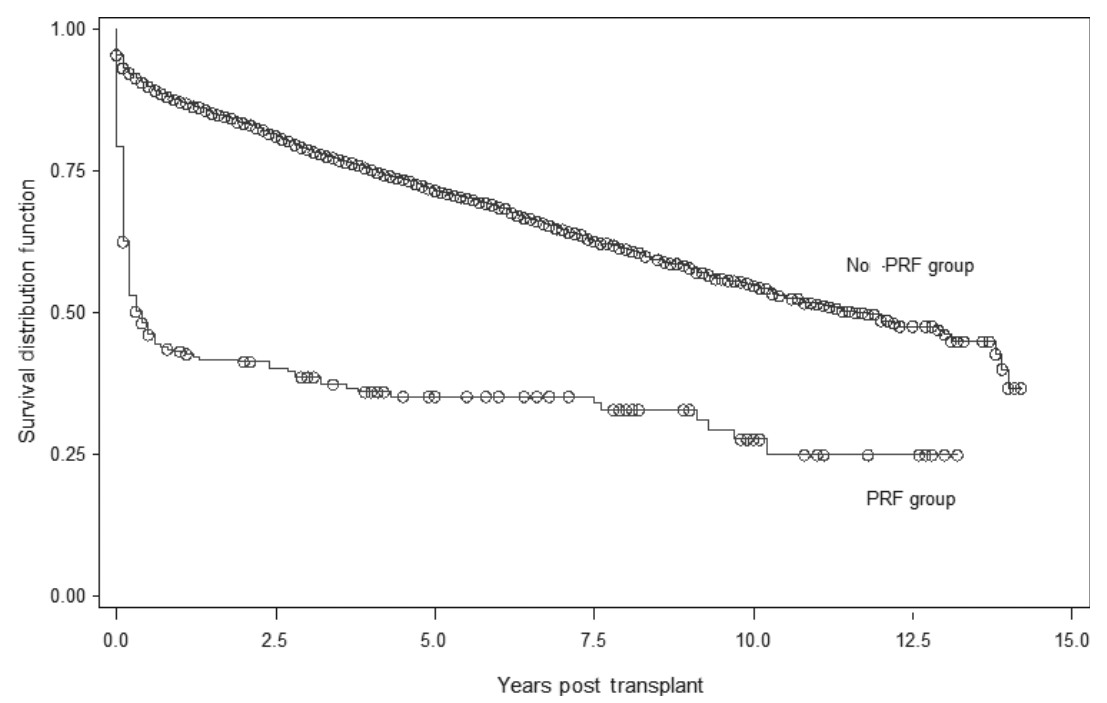

Fig. 3. Graft survival as a function of time post-transplant, stratified by presence or absence of PRF. Survival was significantly lower in the PRF than in the No-PRF group $(\mathrm{p}<0.001)$.

vs. $36 \%$; p $<0.001$ ), and $10 \mathrm{yr}$ post-transplant $(84 \%$ vs. $28 \%$ vs. $35 \%$; p $<0.001)$. For recipients requiring dialysis only pretransplant, survival is similar to the No-PRF group $(\mathrm{p}=0.88)$.

The one, five and 10-yr graft survival was $43 \%, 38 \%$, and $37 \%$ in the PRF group, significantly below the No-PRF group $(88 \%, 79 \%$, and $72 \%$ ), all $p<0.001$ (Fig. 3). The one, five and 10 -yr graft survival was $95 \%, 80 \%$, and $74 \%$ in the PRE group, significantly above the BOTH group $(42 \%, 29 \%$, and $27 \%$; all $p<0.001)$ and POST group $(38 \%, 34 \%$, and $32 \%$; all $\mathrm{p}<0.01)$. For recipients only requiring dialysis pretransplant, graft survival at each interval is similar to the No-PRF group ( $\mathrm{p}=0.88)$.

Independent predictors of death for patients in the PRF group are summarized in Table 3 and include requiring POST, primary diagnosis of CHD, and being of Hispanic ethnicity. Compared with being in the adolescent group, being in the infant group is a predictor of mortality.

Table 3. Risk factors associated with overall mortality in the PRF group

\begin{tabular}{lcc}
\hline Variables & Hazard ratio (95\% Cl) & p Value \\
\hline Univariate & & \\
Serum creatinine level, mg/dL & $1.11(1.01-1.2)$ & $<0.026^{*}$ \\
CHD & $2.2(1.5-3.1)$ & $<0.0001^{*}$ \\
Race (Hispanic vs. Caucasian) & $0.5(0.27-0.95)$ & $0.03^{*}$ \\
POST & $6.0(3.2-8.7)$ & $0.001^{*}$ \\
Multivariate & & \\
CHD & $2.1(1.5-3.1)$ & $<0.0001^{*}$ \\
Race (Caucasian vs. Hispanic) & $0.5(0.27-0.95)$ & $0.031^{*}$ \\
POST & $5.8(3.3-9.6)$ & $0.001^{*}$ \\
\hline
\end{tabular}

Univariate and multivariate risk factors associated with overall mortality in the PRF group using Cox Regression model. CHD represents CHD vs. non-CHD. Asterisk denotes significant difference between groups.
Weight, waiting time, requiring ventilator, inotropic or ECMO support at listing, admission to an ICU pretransplant were not risk factors for mortality in the PRF group.

Morbidity during transplant hospitalization

Transplant hospitalization morbidity is summarized in Table 4 . The length of hospital stay posttransplant was significantly longer in the PRF group than in the No-PRF group. The PRF group experienced more cardiac re-operations, permanent pacemaker implants, significant infections requiring treatment, and stroke than the No-PRF group. There was no difference between groups in the number of rejection episodes before hospital discharge.

Morbidity after hospital discharge

Table 5 summarizes morbidity at one, five, and seven yr post-transplant. Renal dysfunction was

Table 4. Morbidities during transplant hospitalization

\begin{tabular}{lccc}
\hline Parameter & $\begin{array}{c}\text { PRF group } \\
(\mathrm{n}=254)\end{array}$ & $\begin{array}{l}\text { No-PRF group } \\
(\mathrm{n}=3344)\end{array}$ & p Value \\
\hline $\begin{array}{l}\text { Length of hospital stay } \\
\text { (mean } \pm \text { s.d.) }\end{array}$ & $43 \pm 38$ & $24 \pm 40$ & $<0.001$ \\
$\begin{array}{l}\text { Number of acute rejection } \\
\text { episode (mean } \pm \text { s.d.) }\end{array}$ & $0.82 \pm 1.3$ & $0.8 \pm 1.3$ & 0.8 \\
$\begin{array}{l}\text { Cardiac re-operation (\%) } \\
\text { Pacemaker implantation (\%) }\end{array}$ & $63(26)$ & $191(6.3)$ & $<0.001$ \\
$\begin{array}{l}\text { Infection (\%) } \\
\text { Stroke (\%) }\end{array}$ & $153(65)$ & $36(1)$ & 0.003 \\
& $23(9)$ & $70(2)$ & $<0.001$ \\
\hline
\end{tabular}

Morbidity during transplant hospitalization compared between the PRF and No-PRF groups. 
Table 5. Morbidity after hospital discharge one-yr post-transplant

\begin{tabular}{|c|c|c|c|c|c|c|}
\hline & \multicolumn{2}{|l|}{1 -yr post } & \multicolumn{2}{|l|}{5 -yr post } & \multicolumn{2}{|l|}{ 7-yr post } \\
\hline & $\begin{array}{l}\text { PRF } \\
n=113\end{array}$ & $\begin{array}{l}\text { No-PRF } \\
n=2821\end{array}$ & $\begin{array}{l}\text { PRF } \\
n=43\end{array}$ & $\begin{array}{l}\text { No-PRF } \\
n=1458\end{array}$ & $\begin{array}{l}\text { PRF } \\
n=36\end{array}$ & $\begin{array}{l}\text { No-PRF } \\
n=1008\end{array}$ \\
\hline Rejection* & 1.8 & 1.75 & 0.08 & 0.04 & 0.08 & 0.04 \\
\hline $\operatorname{CAD}(\%)^{\dagger}$ & $0(0)$ & $26(0.9)$ & $1(2)$ & $95(6)$ & 1 (3) & $85(8)$ \\
\hline Renal dysfunction (\%) & $23(20)$ & $118(4)$ & $4(8)$ & $117(9)$ & $6(17)$ & 87 (9) \\
\hline Severe renal dysfunction (\%) & $12(11)$ & $26(0.9)$ & $1(2.3)$ & $14(1)$ & $2(5.5)$ & $19(2)$ \\
\hline Chronic dialysis & 7 & 9 & 1 & 5 & 1 & 7 \\
\hline Serum creatinine $>2.5$ & 12 & 25 & 1 & 14 & 2 & 17 \\
\hline Renal transplant & 2 & 0 & 1 & 1 & 0 & 2 \\
\hline Infection (\%) & $41(36)$ & $711(25)$ & $7(16)$ & $172(12)$ & 5 (14) & $91(9)$ \\
\hline Diabetes (\%) & $3(3)$ & $55(2)$ & $1(2)$ & $35(4)$ & $4(12)$ & $23(2)$ \\
\hline Hypertension (\%) & $32(28)$ & $588(20)$ & $20(47)$ & $506(35)$ & $19(57)$ & 344 (37) \\
\hline
\end{tabular}

Mean episode of rejection, infection-requiring hospitalization, incidence of CAD, renal dysfunction, diabetes, and hypertension compared between PRF and No-PRF group at one, five, and seven yr post-transplant.

${ }^{*}$ Mean episode of rejection.

†Number and percentage of coronary artery disease.

more common in the PRF group than in the No-PRF one-yr post-transplant $(\mathrm{p}<0.001)$, but this difference did not persist at five and seven yr post-transplant. Severe renal dysfunction, defined as requiring chronic dialysis, renal transplant or with a serum creatinine concentration above $2.5 \mathrm{mg} / \mathrm{dL}$, was significantly more prevalent in the PRF group than in the No-PRF at one-yr post-transplant. As with renal dysfunction, the difference in incidence of severe renal dysfunction did not persist at five and seven yr. Of recipients in the PRF group, nine died within five $\mathrm{yr}$ post-transplant and the other three recipients recovered their renal function. Twenty-six $(1 \%)$ recipients in the No-PRF group had severe renal dysfunction at one yr post-transplant, of whom 15 died within five yr, six recovered renal function, and five persisted with renal dysfunction at most recent follow up. The prevalence of diabetes and hypertension was higher in the PRF group than in the No-PRF group only at seven $y r$ post-transplant $(\mathrm{p}=0.003$ and $\mathrm{p}=0.04)$. The incidence of CAD, the number of rejection episodes and infections requiring hospitalization did not differ between the PRF and No-PRF groups at any time posttransplant.

Risk factors associated with PRF

Univariate analysis of variables identified seven significant risk factors for developing PRF as shown in Table 6. Risk factors included requiring ECMO, ventilator, or inotropic support at listing, having an infection requiring intravenous drug therapy within two wk prior to listing, $\mathrm{CHD}$ as the listing diagnosis, ICU admission vs.
Table 6. Risk factors for developing PRF

\begin{tabular}{llc}
\hline Variables & Relative risk (95\% CI) & p Value \\
\hline Univariate & & \\
ECMO support (Y) & $5.9(3.9-7.9)$ & $<0.0001$ \\
Ventilator support (Y) & $2.6(2.0-3.5)$ & $<0.0001$ \\
Infection* & $2.3(1.5-3.6)$ & 0.0027 \\
CHD* & $2.1(1.6-2.7)$ & 0.003 \\
Medical condition (ICU vs. home) & $2.1(1.5-2.9)$ & $<0.0001$ \\
Inotrope support (Y) & $1.6(1.2-2.1)$ & $<0.0001$ \\
Multivariate & & \\
ECMO support (Y) & $3.1(2.04 .9)$ & $<0.0001$ \\
Ventilator support (Y) & $1.9(1.3-2.7)$ & 0.0006 \\
CHD* & $2.1(21.5-2.8)$ & $<0.0001$ \\
Medical condition (ICU vs. home) & $1.1(1.1-2.4)$ & $<0.0001$ \\
\end{tabular}

Pretransplant risk factors for developing PRF obtained by univaraite and multivariate regression models. Infection $\left({ }^{*}\right)$ represents significant infection requiring intravenous treatment two wk prior to listing. Medical condition represents recipient stay in ICU as opposed to home at the time of listing. ECMO, ventilator, or inotrope support refer to support at the time of listing.

being home at the time of listing. In multivariate analysis, risk factors that remained significant included requiring ECMO and ventilator support, primary diagnosis of CHD and ICU stay at the time of listing for transplant.

\section{Discussion}

This study represents the largest analysis of PRF in the pediatric heart transplant experience. There is a paucity of data on the impact of PRF on transplant outcomes from any single institution because of the rarity of heart transplantation in children. To overcome this limitation, the UNOS/OPTN registry was used to assess outcome in primary heart transplant recipients. Our study found that the prevalence of PRF was 7\% 
in pediatric primary heart transplants performed between 1993 and 2008. Post-transplant patient survival was only compromised in patients who required dialysis post-transplant (whether or not it began pretransplant. PRE need only did not impact short- and long-term outcomes. When patient survival was made conditional upon surviving to six months post-transplant, the difference between the PRF and No-PRF groups became insignificant. In our study, requiring POST, a primary diagnosis of CHD and being of Hispanic ethnicity were independent predictors of mortality in the PRF group.

PRF is experienced by $11-15 \%$ of adult heart transplant recipients (5), and is a major determinant of one-yr survival and subsequent chronic renal failure (4-8). Information on PRF in pediatric heart transplant recipients is limited. A single center study reported that $12.6 \%$ of 308 pediatric transplant recipients developed PRF, and had lower one and five-yr survival than No-PRF patients (10).

The present report has advantages over single center reports in that it captures data from all children who received a heart transplant in the United States, increasing statistical power to identify several important national trends. Specifically, it is the first study to our knowledge that defines the prevalence of PRF in pediatric cardiac transplant recipients, determines its impact on heart transplant outcome and identifies risk factors associated with developing PRF. We found a lower prevalence of PRF $(7 \%)$ than the adult study (5), likely because pediatric recipients generally have a lower incidence of comorbidities than adults. We also found a better post-transplant survival than that reported from a single pediatric transplant center (10), likely because death of a few patients from a single center study will skew outcome data negatively.

PRF is a well-known complication after cardiopulmonary bypass. The incidence of PRF in children with normal kidney function undergoing open heart surgery is reported to range from $2 \%$ to $11 \%(11-13)$. The incidence increased to $25 \%$ in children with solitary functioning kidney (14). Requiring renal placement therapy post-heart surgery carries as high as $40-80 \%$ of mortality $(14,15)$. Our study found that the incidence of PRF and mortality in pediatric heart transplant recipients were similar to children undergoing non-transplant open heart surgery.

Compared with transplant recipients with a primary diagnosis of cardiomyopathy, those with a primary diagnosis of CHD are at increased risk for developing PRF. The most significant impact of PRF on survival of pediatric cardiac transplant is seen during the first six months after surgery.

There is huge difference in post-transplant patient survival between PRE and POST groups. The one, five and 10-yr graft survival is $95 \%$, $80 \%$, and $74 \%$ in the PRE group, significantly above POST group $(38 \%, 34 \%$, and $32 \%$; all $\mathrm{p}<0.01)$. An explanation for this could be that PRE is most likely secondary to heart failure and improvement of cardiac function post-transplant provides an opportunity to recover renal function. By contrast, PRF that included the need for dialysis post-transplant had negative impact on survival. This may relate to poor graft function on other comorbidity that produces permanent renal dysfunction.

In our study, $40 \%$ of recipients requiring PRE did not require POST and they had an excellent outcome, which suggests that patients needing dialysis while awaiting a heart transplant should be considered suitable heart transplant candidates.

The risk factors associated with developing PRF identified in our study can be applied clinically. For these high-risk patients, it is particularly important to avoid nephrotoxic agents while awaiting heart transplant, to optimize peri-operative hemodynamic status to prevent prolonged low cardiac output and multiple organ failure. Strategies to optimize hemodynamics include carefully maintain euvolemia, judicious use of inotropic support, prophylactic administration of renal protection strategies such as dopamine infusion, and consideration of mechanical support.

\section{Limitations}

This is a multi-institutional retrospective database study which limits the strengths of conclusions derived by pooling institutional experiences that have different treatment protocols for their patients both pre- and post-transplant. This database does not allow us to assess the effect of type of CHD (HLHS, single ventricle, and two ventricle), prior heart surgery (failed palliation and rescue transplant surgery), or duration of renal replacement support on outcomes. We were not able to take into account the immunosuppressive regimen on renal dysfunction because of database limitations. There are only 254 patients in the PRF group. Such a small number of patients do not allow us to identify black race as a risk factor for mortality or examine the additive effect of risk factors such as combined CHD and ECMO or ventilator support. 
Tang et al.

\section{Disclosure}

The authors have no financial conflict of interest to disclose.

\section{References}

1. De Jonge N, Kirkels JH, Klöpping C, et al. Guidelines for heart transplantation. Neth Heart J 2008: 16: 79-87.

2. Boucek MM, Aurora P, Edwards LB, et al. Registry of the International Society for Heart and Lung Transplantation: Tenth official pediatric heart transplantation report - 2007. J Heart Lung Transplant 2007: 26: 796-807.

3. Cantarovich M, Hirsh A, Alam A, et al. The clinical impact of an early decline in kidney function in patients following heart transplantation. Am J Transplant. 2009: 9: 348-354 [Epub ahead of print].

4. Odim J, Wheat J, Lakis $\mathrm{H}$, et al. Peri-operative renal function and outcome after orthotopic heart transplantation. J Heart Lung Transplant 2006: 25: 162-166.

5. Meyer SR, Modry DL, Norris CM, et al. Pretransplant diabetes, not donor age, predicts long-term outcomes in cardiac transplantation. J Card Surg 2006: 21: 117-124.

6. Greenberg A, Thompson ME, Griffith BJ, et al. Cyclosporine nephrotoxicity in cardiac allograft patients - A sevenyear follow-up. Transplantation 1990: 50: 589-593.

7. Vossler MR, Ni H, Toy W, Hershberger RE. Pre-operative renal function predicts development of chronic renal insufficiency after orthotopic heart transplantation. J Heart Lung Transplant 2002: 21: 874-881.
8. Lee CK, Christensen Ll, Magee JC, Ojo AO, Harmon We, BRIDGES ND. Pre-transplant risk factors for chronic renal dysfunction after pediatric heart transplantation: A 10-year national cohort study. J Heart Lung Transplant 2007: 26: 1077.

9. SAHNey S, ChinNock R. Management of infants and young children with combined heart and kidney failure. Pediatr Transplant 2006: 10: 408-412.

10. Jacobs JP, Quintessenza JA, Chai PJ, et al. Rescue cardiac transplantation for failing staged palliation in patients with hypoplastic left heart syndrome. Cardiol Young 2006: 16: 556562.

11. Baxter P, Rigby M, Jones O, Lincolen C, Shinebourne E. Acute renal failure after cardiopulmonary bypass in children: Results of treatment. Int J Cardiol 1985: 7: 235-239.

12. SkipPen PW, Krahn GE. Acute renal failure in children undergoing cardiopulmonary bypass. Crit Care Resusc 2005: 7: 286-291.

13. Авоu El-Ella RS, Najm HK, Godman M, Kabbani MS. Acute renal failure and outcome of children with solitary kidney undergoing cardiac surgery. Pediatr Cardiol 2008: 29: 614618. Epub 2007 Dec 15.

14. Boigner H, Brannath W, Hermon M, et al. Predictors of mortality at initiation of peritoneal dialysis in children after cardiac surgery. Ann Thorac Surg 2004: 77: 61-65.

15. Picca S, Principato F, Mazzera E, et al. Risks of acute renal failure after cardiopulmonary bypass surgery in children: A retrospective 10-year case-control study. Nephrol Dial Transplant 1995: 10: 630-636. 